\title{
Importance of Interfacial Interactions to Access Shear Elasticity of Liquids and Understand Flow Induced Birefringence from Liquid Crystals to Worm-Like Micellar Solutions
}

\author{
Laurence Noirez ${ }^{*}$ \\ Laboratoire Léon Brillouin (CEA-CNRS), Université Paris-Saclay, CEA-Saclay, 91191 Gif-sur-Yvette Cedex - France \\ e-mail: laurence.noirez@cea.fr \\ * Corresponding author
}

\begin{abstract}
This work points out the importance of the substrate boundary conditions to lower the dissipation in the dynamic measurement and access the closest dynamic characteristics of liquids, in particular to access the low frequency shear elasticity. The liquid/surface interface is a source of dissipation that enters and impacts the measurement. Examples of steady-state shear flows or flow birefringence are presented to highlight the non-universality of the behavior with respect to the nature of the substrate or the sheared thickness. Additionally the present development completes and extends the identification of low frequency shear elasticity made at sub-millimeter gaps in various one-component liquids to salt-free aqueous solutions (CTAB-water (Hexadecyl-TrimethylAmmonium Bromide)).
\end{abstract}

Résumé - Importance des interactions interfaciales pour accéder à l'élasticité de cisaillement des liquides et comprendre la biréfringence d'écoulement des cristaux liquides aux solutions micellaires - Nous montrons par une approche expérimentale, l'importance de l'interaction liquide/substrat (mouillage) pour diminuer la dissipation et améliorer la mesure dynamique, en particulier pour accéder à l'élasticité de cisaillement à basse fréquence peu accessible par la mesure viscoélastique conventionnelle. L'interface liquide/surface est une source de dissipation qui a un impact dans la mesure. À titre d'exemple, des mesures d'écoulement en cisaillement continu ou de biréfringence d'écoulement sont présentées pour mettre en évidence la non-universalité du comportement par rapport à la nature du substrat ou à l'épaisseur cisaillée. Enfin, nous étendons la mesure de l'élasticité de cisaillement basse fréquence réalisée à l'échelle sub-millimétrique dans les liquides purs à des solutions lyotropes de micelles géantes (CTAB-water (HexadecylTrimethylAmmonium Bromide)). 


\section{INTRODUCTION}

Among the most spectacular liquid behaviors is the ability to produce birefringence under flow. This observation dates back from the early $\mathrm{XX}^{\text {th }}$ century with the huge (172 fluids) and pioneering work of Vorländer and Walter in 1925 [1], followed by the outstanding work from Sadron in 1936 highlighting shear bands [2] or the systematic study of alkanes by Champion and North in 1968 [3]. They prove that the wide majority of pure liquids exhibit birefringence and shear bands when submitted to very high shear rates (Fig. 1).

Much later the flow birefringence is again in the spotlight with the identification of shear induced phase transitions in aqueous solutions of worm-like micelles [4-7]. The birefringence is observed at relatively low shear rates and occurs above a shear threshold. Its origin is discussed at length on the basis of a coupling with pre-transitional orientational life time $\tau[8,9]$ in analogy to the coupling model very early proposed by Hess [10] and then by Olmsted and Goldbart [11], predicting non-equilibrium transitions in the liquid phase of thermotropic liquid crystals. While a wide literature that (we do not here develop), exists on rheo-optical behavior of lyotropic solutions, much less concerns thermotropic systems, probably because their experimental study requires highly accurate controlled temperature environments. Reversible non-equilibrium transitions in the isotropic phase of thermotropic systems were however clearly established on side-chain liquid crystal polymers including low molecular species [12]. Here again, the birefringence is easily induced above a very low shear rates (of the order of $\mathrm{s}^{-1}$ ). This property is identified regardless the molecular weight or the chemical formula in the liquid (isotropic) phase liquid crystalline polymers depicting a generic property [12].

The temperature in thermotropic liquid crystals plays an equivalent role as the concentration in lyotropic worm-like micellar solutions and leads under flow the induction of birefringence in the liquid phase. But thermotropic systems have a certain advantage over the lyotropic solutions, in that the time of pre-transitional fluctuations $\tau$ is known and enables to test the validity of the theoretical interpretations in terms of coupling with pretransitional fluctuations [11]. The confrontation to the timescale of the pretransitional fluctuations reveals that the shear rates at the origin of the birefringence do not match any known molecular relaxation time (a flow coupling is expected when the shear rate is of the order of the inverse of the longest characteristic time (following the Deborah relationship: $\tau \dot{\gamma}>1)$ ). The theoretical approaches based on a timescale coupling are invalidated opening new research routes. The present paper gathers some of these routes, highlighting few experimental results showing the influence of external parameters as the surface boundary conditions in the flow behavior, the flow birefringence or the dynamic relaxation. This short letter contributes to point out the role of the liquid/surface interface to better understand the liquid state and take into account its shear elasticity.

\section{KEY ROLE OF THE SURFACE IN THE FLOW BEHAVIOR: SOME EXAMPLES FROM FLOW BIREFRINGENCE AND SHEAR STRESS MEASUREMENTS}

While the literature continuously reports on new developments related to flow behavior and properties, very few studies focus on the impact of surface/liquid boundary conditions. The flow analysis in rheology is however entirely related on the transmission of the motion via the substrates. Figure 2 illustrates the chain measurement of the shear stress in (linear and non-linear) rheology.

A second interface plays a crucial in the transmission of the stress: the air/liquid interface. Microscopic observation indicates that most of the liquids contain microbubbles, cavities, invisible to the naked eye (Fig. 3a). These insertions can significantly damp the transmission of the mechanical stress in the liquid bulk but also by the aggregation of nanobubbles at the surface forming micropancakes. The consequences in the stress measurement under flow are a noisy signal, a slippage state, an impossibility to have a linear response and wrong interpretations of a flow response. Figures $3 \mathrm{~b}$ and $3 \mathrm{c}$ illustrate the change in appearance obtained prior and after simple degassing on an industrial sample of polybutadiene (degassing).

In the same way that nanobubbles at the surface lowers the contacts of the molecules to the surface, the force of the molecular interactions to the surface determines the macroscopic flow behavior. Liquids as the liquid phase of liquid crystals are excellent probe to demonstrate that steady-state flows are dependent on external parameters as the surface. Figures $4 \mathrm{a}$ and $4 \mathrm{~b}$ indicate a non-universal rheological response in a liquid state, expected to exhibit a viscous behavior while the experimental result points out the occurrence of slippage transitions at the surface [13].

\section{THE KEY ROLE OF THE SURFACE IN DYNAMIC RELAXATION MEASUREMENTS}

In contrast to flow experiments, dynamic relaxation (also called dynamic mechanical analysis) aims at probing the properties in nearly equilibrium conditions (linear conditions). The interaction to the surface is also crucial in this measurement. Dynamic relaxation is employed to determine the viscous or solid-like nature of a material deduced from its stress response to a low frequency shear solicitation. This method consists in transmitting via the surface contacts 


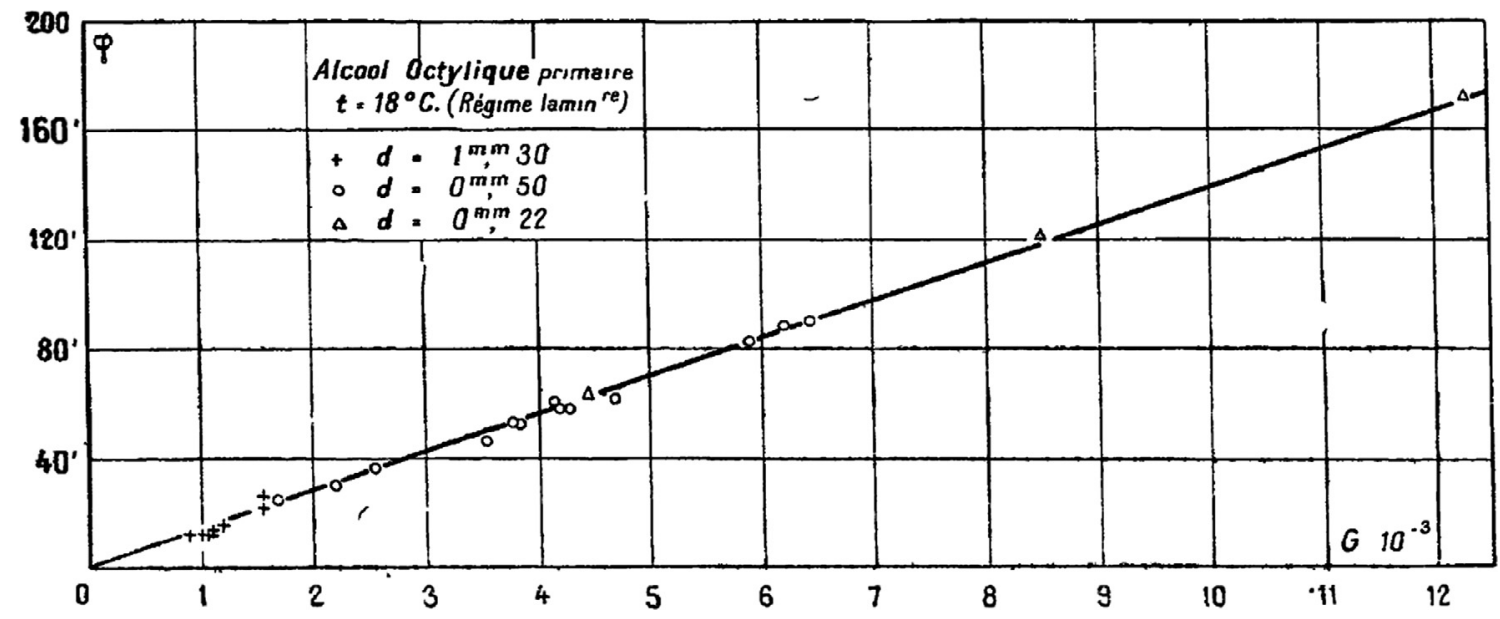

Figure 1

Flow birefringence and shear bands were early identified in various liquids as illustrated here by the birefringence induced in ethanol under high shear flow carried out in 1936 by C. Sadron [2].

(the geometry of the setup is illustrated in Fig. 2), a low amplitude oscillatory shear strain to probe equilibrium properties of the sample. The equilibrium state properties are typically described in the frequency $(\omega)$ range from $0.01 \mathrm{rad} \mathrm{s}^{-1}-100 \mathrm{rad} \mathrm{s}^{-1}$.

The analysis of the signal defines the strain range for which the conventional formalism in terms of elastic $G^{\prime}$ and viscous $G^{\prime \prime}$ moduli can be applied (both imposed strain wave and measured stress wave exhibit the same harmonic (sinusoidal-like)). Under these conditions, shear stress and shear strain are related by: $\sigma(\omega)=G_{0} \gamma_{0} \sin (\omega t+\Delta \phi)$ with $G_{0}$ the viscoelastic modulus, $t$ the time, $\omega$ the frequency and $\Delta \phi$ the phase shift between the input and the output waves, or in terms of shear elastic $\left(G^{\prime}\right)$ and viscous $\left(G^{\prime \prime}\right)$ moduli: $\sigma(\omega)=\gamma_{0}\left(G^{\prime}(\omega) \sin (\omega t)+G^{\prime \prime}(\omega) \cos (\omega t)\right)$, with $G^{\prime}$ the component in phase with the strain, and $G^{\prime \prime}$ the out of phase component.

A liquid-like character is identifiable by a vanishing response at long time scale whereas a solid-like behavior (elastic) exhibits a finite response independent of the frequency (Fig. 5a). When the phase shift is lower than $\Delta \phi<\pi / 4$, the elastic component $G^{\prime}$ dominates the viscous component $G^{\prime \prime}$ (Fig. 1 right). If the output response of the material presents a phase shift larger than $\pi / 4$, then the viscous component $G^{\prime \prime}$ dominates the elastic component $G^{\prime}$ (Fig. 5b) and the behavior is called liquid-like. If the phase shift reaches $\pi / 2$ ( $G^{\prime}$ vanishes), the sample is defined as purely viscous. A (Maxwell) liquid-like behavior displays typically a vanishing response characterised by a $\omega$-scaling of the viscous modulus and a $\omega^{2}$-scaling dependence of the elastic modulus (Fig. 5c).

Recent experimental developments have shown that reinforcing the surface contacts using high energy surface

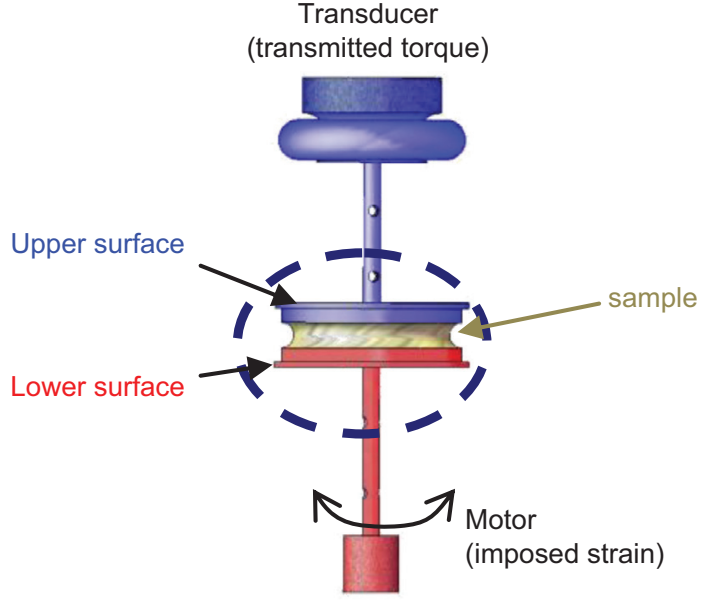

Figure 2

The surface interaction governs the transmission chain in rheology measurements: the stress is transmitted to the molecules interacting with the first surface fixed to the motor, is communicated along the sample thickness and is transferred via molecular contacts to the second surface coupled to a force (here a torque) sensor.

improves the measurement of the dynamic response [14-21]. When the liquid molecules are strongly in interaction with the surface, the noise observable at low frequency grows and forms a well-defined signal, stronger than the viscous response (Fig. 5c). The resulting signal reveals instead of a flow behavior, an elastic behavior particularly visible at the submillimeter thickness, typically $0.100-0.500 \mathrm{~mm}$ in various liquids (Fig. 6).

Shear elasticity means that the liquid molecules are long range elastically correlated (within the considered frequency 


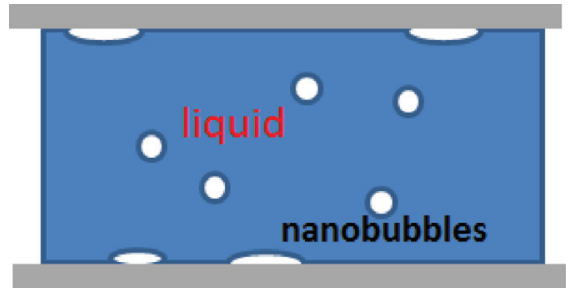

a)

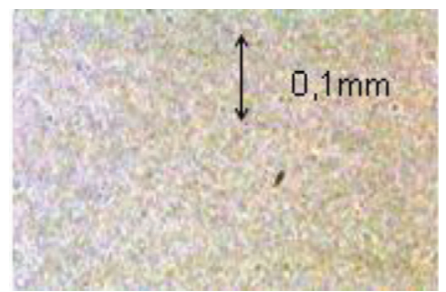

b)

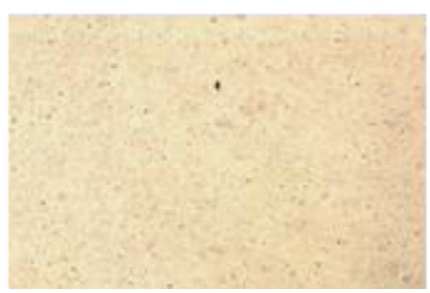

c)

Figure 3

a) Scheme of a liquid layer with micro/nanobubbles in the bulk and at the interfaces, b) microscopic photographs of a polybutadiene bulk prior and c) after degassing. Without degassing, the polymer exhibits a conventional viscoelastic behavior while after degassing, it is possible to reveal a low frequency elastic behavior.

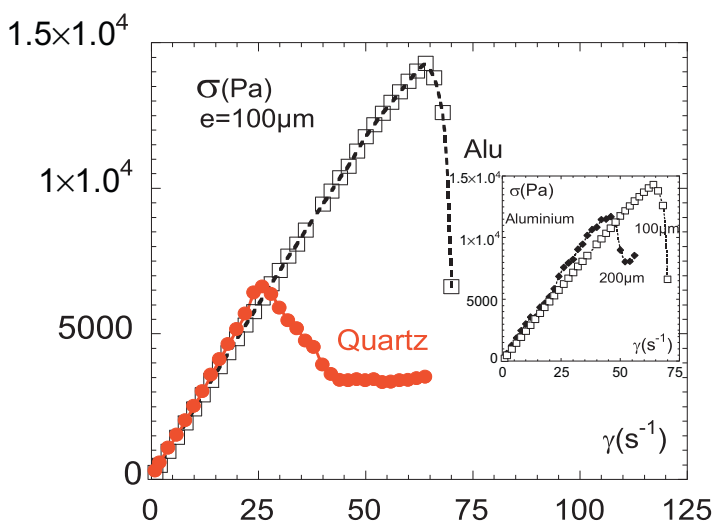

a)

Figure 4

External parameters as the surface impact the flow. This is particularly visible at the sub-millimeter scale. The figures illustrate steady state flow behavior in the liquid (isotropic) phase of a low molecular weight liquid-crystal polymer (generic behavior) from [12]: a) shear stress versus shear rate measured on different substrates. In the Newtonian regime, shear stress and shear rate are proportional $\sigma=\eta \dot{\gamma}$ where $\eta$ is the viscosity: red $(100 \mu \mathrm{m})$ : quartz substrate, $\square(100 \mu \mathrm{m})$ : aluminium substrate, at low shear rates $\left(\dot{\gamma}<40 \mathrm{~s}^{-1}\right.$ for the quartz and $70 \mathrm{~s}^{-1}$ for the aluminium substrate), the flow is linear to the shear rate (Newtonian regime). At higher shear rates, the departure from the linearity indicates a slippage transition which is a function of the force of the interactions to the substrate. In the inset, the shear stress versus shear rate is reported at $200 \mu \mathrm{m}: \Delta$, and at $100 \mu \mathrm{m}: \square$ (aluminium substrate). b) Flow birefringence $<\Delta n_{x}>$ versus shear rate at different gap thicknesses: (blue $\left.\bigcirc\right) 75 \mu \mathrm{m}$, (red $\left.\bullet\right) 100 \mu \mathrm{m}$ and (green $\triangle$ ) $125 \mu \mathrm{m}$. Similarly, the occurrence of the birefringence plateau at high shear rates indicates a slippage at the surface. This slippage regime at constant shear rate depends on the sheared thickness as here illustrated.

range) implying that intermolecular interactions play a key role in the flow process. These long-range interactions are absent in molecular theories (theory of viscoelasticity).

We show here by improving the liquid/substrate contact that it is possible to extend the measurement of the low frequency shear elasticity to solutions (binary systems). For this study a specific chamber has been built to avoid hygroscopic changes and saturate the surrounding air in water vapour. In contrast to one-component liquids, two-component liquids as micellar solutions requires a specific care because of possible migration of solute to the surfaces of the substrate and/or on at the air/liquid interface inducing non-homogeneities at the boundaries that might false the stress measurement. The following measurements are thus carried out relatively rapidly, made under controlled atmosphere, at narrow gap (typically $0.100,0.200$ or $0.300 \mathrm{~mm}$ gap thickness) and the reproducibility is checked by scanning from high to low and from low to high frequencies to guarantee that the sample does not have evolved during the measuring time (Fig. 7b). The chosen aqueous surfactant solution is the salt-free CTAB-water (Hexadecyl-TrimethylAmmonium Bromide) diluted at 


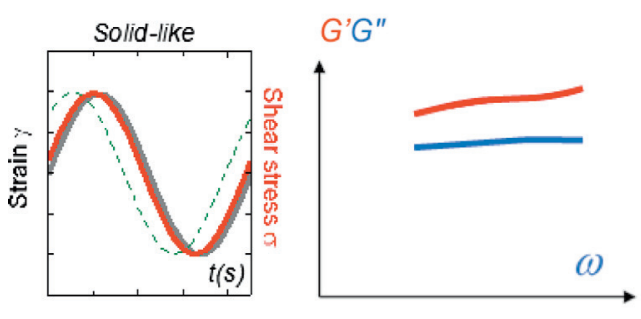

a)

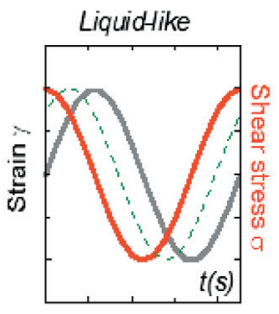

b)
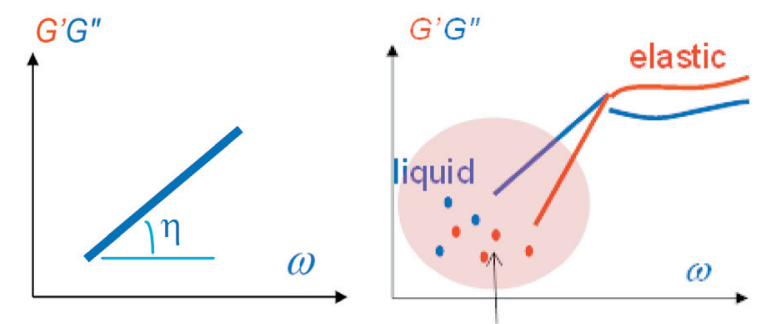

c) Iow frequency noise

Figure 5

Schemes of the sin strain input wave (-) and shear stress output response (red - ) (the dotted line represents the $\pi / 4$ phase shift output wave (- - )), and corresponding low frequency dynamic spectra (logarithmic representation). a) Solid-like behavior: input and output waves are close in phase: $G^{\prime}>G^{\prime \prime}$ with $G^{\prime}$ and $G^{\prime \prime}$ weakly dependent on the frequency. b) Viscous behavior: input and output waves are out of phase with $\Delta \phi>\pi / 4$ (here $\Delta \phi=\pi / 2$ ) with $G^{\prime}$ negligible and $G^{\prime \prime}$ vanishing with the frequency. c) Improving liquid/surface interactions reduces the dissipation, suppresses the low frequency noise and makes appear an elastic response (Fig. 5a).
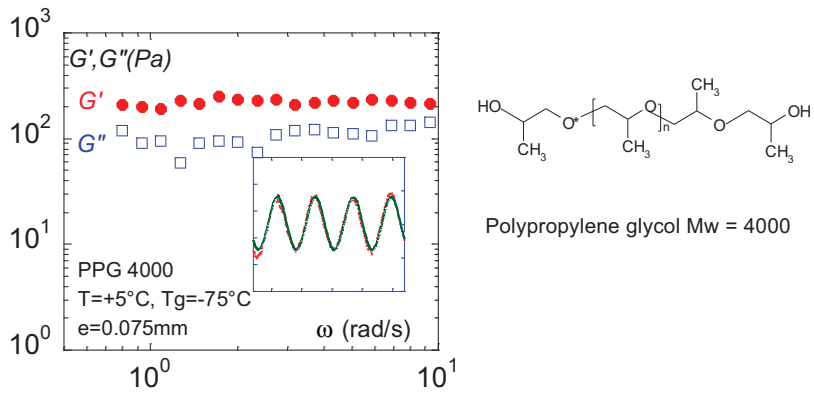

Polypropylene glycol $\mathrm{Mw}=4000$

Figure 6

The transfer of the motion to the liquid is here achieved by ensuring total wetting boundary conditions between the liquid and the substrate ( $\alpha$-alumina plates) and by probing small gaps. Improving the forces of the interactions between the liquid and the substrate surface (total wetting conditions) enables to access low frequency shear elasticity in a wide panel of liquids at the sub-millimeter scale. Frequency dependence of the elastic $G^{\prime}(\omega)$ : red $\bigcirc$ and viscous moduli $G^{\prime \prime}(\omega)$ : blue $\square$, measured for a glass former liquid (Polypropyleneglycol - PPG4000, $\left.\mathrm{Mw}=4000, T_{\mathrm{g}}=-75^{\circ} \mathrm{C}\right)$ at $T=+5^{\circ} \mathrm{C}(0.075 \mathrm{~mm}$ gap thickness - alumina plate-plate fixtures). The insert shows the superposition of the strain (green points) and the stress (red points) waves highlighting the instant response of the liquid [14].

$18 \%$ and measured at $33{ }^{\circ} \mathrm{C}$. This surfactant solution is used as antiseptic agent against bacteria and fungi. It has been widely studied from a rheological (linear and non-linear) point of view (while not well understood). It exhibits an isotropic-nematic/hexagonal phase diagram by increasing the surfactant concentration that is dependent on the temperature (inset Fig. 7 from [22]). The chosen concentration $(18 \%)$ and temperature $\left(T=33{ }^{\circ} \mathrm{C}\right)$ correspond to the isotropic phase of the phase diagram. In this isotropic phase, the conventional dynamic relaxation measurement carried on aluminium substrate displays a typical Maxwell behavior indicating a viscoelastic fluid. Similarly as for thermotropic liquid crystals (Fig. 4), the isotropic phase displays a nonlinear rheological behavior with a discontinuous variation of the shear stress with the shear rate, and optically the emergence of birefringent bands above a critical shear rate, indicating that the isotropic liquid transits from an isotropic state to a birefringent state assimilated to a shear induced isotropic-nematic phase transition.

The identification of low frequency shear elasticity in the liquid phase of thermotropic systems as here illustrated in Figure $7 \mathrm{a}$ is interpreted as the result of the solicitation of elastic correlations $[17,19,23]$. Since the isotropic phase of liquid crystal polymers is the thermotropic equivalent of the isotropic phase of lyotropic solutions and since shear elasticity was identified in the liquid phase of liquid crystals [17-21], we show here that it is also possible to access low frequency shear elasticity in worm-like micellar solutions (Fig. 7b) bringing possible new input in the long debate on the origin of shear induced phase transitions in lyotropic solutions. Figure $7 \mathrm{~b}$ displays the low frequency behavior in the isotropic phase of CTAB-Water. Similarly as for the isotropic phase of the thermotropic system, the elastic modulus $\left(G^{\prime}\right)$ is higher than the viscous modulus $\left(G^{\prime \prime}\right)$ with $G^{\prime}$ and $G^{\prime \prime}$ relatively independent of the frequency, indicating a dominant elastic response.

The identification of shear elasticity in both thermotropic and lyotropic systems indicates that the intermolecular interactions are extended at long range. They originate from electrostatic interactions between molecules that provide the liquid cohesion. The use of strongly interacting surface to characterize the dynamic behavior of surfactant solution (Fig. 7b) indicates a low frequency elastic-like behavior (shear modulus and viscous modulus are independent of the frequency with $G^{\prime}>G^{\prime \prime}$ ), and thus long range elastic correlations in agreement with what was found on various liquids including ionic liquids, alkanes, liquid water $[15,19,24]$. 
Isotropic phase of Thermotropic Liquid Crystal Polymer

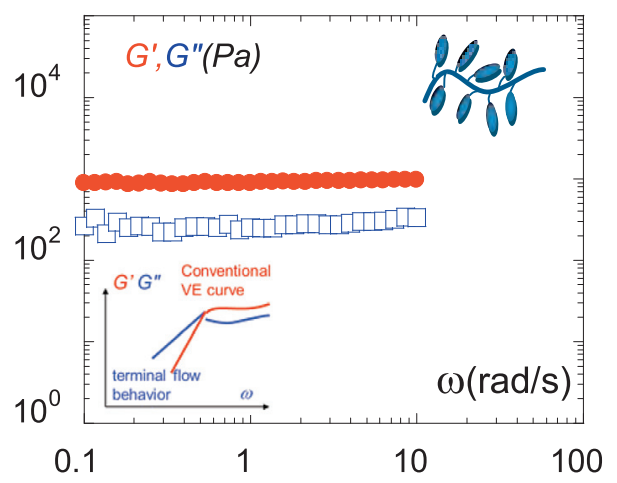

Isotropic phase of Lyotropic solution $\left(\mathrm{CTAB} 18 \% \mathrm{~T}=33^{\circ} \mathrm{C}\right)$

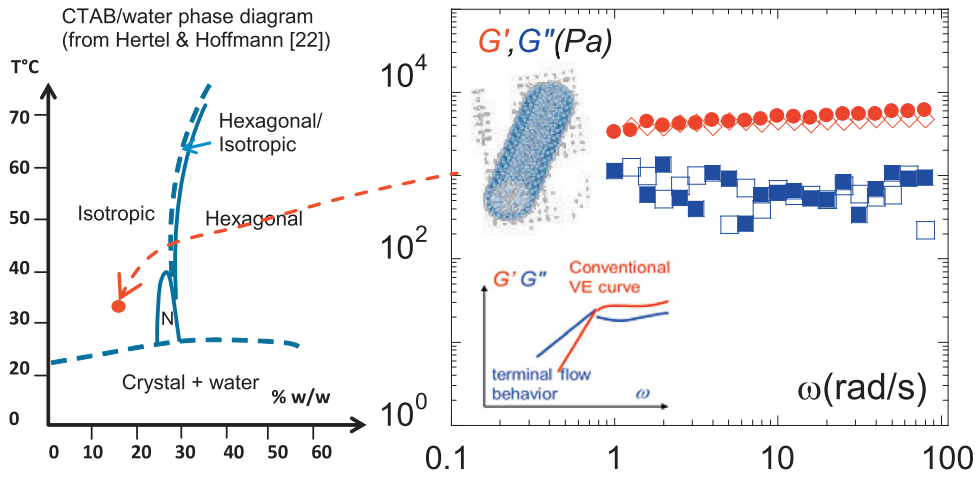

Figure 7

By improving the interactions to the surface, the dynamic measurement reveals a solid-like response $\left(G^{\prime}(\right.$ red $\left.\bullet)>G^{\prime \prime}(\diamond)\right)$ in: a) thermotropic liquid crystal: the liquid phase (here at $14{ }^{\circ} \mathrm{C}$ above the isotropic-nematic transition and at $0.150 \mathrm{~mm}$ thickness) of a liquid-crystal polymer. The inset displays for comparison the conventional flow behavior [as predicted for uncross-linked polymers (Maxwell model)] [21, 25]. Thermotropic liquid crystal I(isotropic) $-119^{\circ} \mathrm{C}-\mathrm{N}$ (nematic) $-30{ }^{\circ} \mathrm{C}-$ Glassy state. b) CTAB: Phase diagram from reference [22]. Lyotropic liquid crystal: the liquid phase of salt-free CTAB-water (dilution $18 \%$ ) measured at $33{ }^{\circ} \mathrm{C}$ at $0.2 \%$ strain amplitude, $0.3 \mathrm{~mm}$ gap thickness. The shear modulus and the viscous modulus are independent of the frequency with $G^{\prime}>G^{\prime \prime}$ indicating a solid-like behavior. Similar responses are obtained by scanning from low to high $G^{\prime}(\mathrm{Pa})$ : red $\bullet, G^{\prime \prime}(\mathrm{Pa})$ : blue $\mathbf{\square}$ and from high to low frequency $G^{\prime}(\mathrm{Pa})$ : red $\diamond, G^{\prime \prime}(\mathrm{Pa})$ : blue $\square$.

\section{CONCLUSIONS}

The few examples shown here illustrate how much the molecular coupling to the surface (via wetting, adhesion, and other interfacial mechanisms) determines the flow behavior even at a macroscopic scale. The liquid/surface interface is a source of complex dissipation mechanisms as partial slippage at the wall. Resulting non-linear states are been experimentally identified in steady-state shear flows in both entangled and non-entangled polymer melts [26], microgel suspensions [27] and even locally on grafted polymer brushes [28] and theoretically very early predicted in microfluidics [29].

By reinforcing the interactions to the solid substrate, it is possible to probe more efficiently the mechanical response of liquids to a dynamic shear wave and access the complete dynamic description of the fluid. Prior measurements have shown that these conditions are required to measure the shear elasticity of liquids [14, 18 and references therein]. Here in addition, we show that the low frequency shear elasticity identified in various one-component liquids, is also detectable in a salt-free surfactant solution response at submillimeter scale in solutions confirming its generic intermolecular origin. This was made possible using high energy surfaces as $\alpha$-alumina in replacement of conventional metallic substrates (made of aluminum, stainless steel) that provides a partial wetting only. The force of the interfacial liquid/substrate interactions is thus essential to lower the interfacial dissipation during the dynamic measurement and access the full dynamic characteristics of the sample revealing the liquid elasticity.
The shear elasticity at low frequency is of first importance as it challenges the molecular approaches of physics of liquids. This experimental approach of liquids might join recent theoretical developments on the fusion of amorphous solids [30], simulations revisiting the liquid state introducing flexible bond-forming liquids in various liquids [31] or predicting the elasticity, at several molecular lengths scale from the surface [32]. The existence of shear elasticity in liquids was remarkably foreseen about 20 years ago by Volino using a non-extensive approach [33]. However much remains to be made to understand the interfacial mechanisms that relate shear elasticity and surface interactions. The challenge is ambitious involving every aspect of dynamic processes from physical, chemical to biological processes [34] but makes also possible the emergence of new effects as for example the low frequency elastic birefringence [35].

\section{ACKNOWLEDGMENTS}

The author is particularly grateful to her co-workers, Philipp Kahl, Hakima Mendil-Jakani and in particular Patrick Baroni for the numerous instrumental innovations and to TA-Instruments and $R \& D$ Vision for providing valuable scientific equipment.

\section{REFERENCES}

1 Vorländer D., Walter R. (1925) The mechanically produced double refraction of amorphous liquids and its connection with molecular form, Z. Phys. Chem. 118, 1-30. 
2 Sadron C. (1936) Sur la birefringence dynamique des liquides purs, J. Phys. Radium 7, 263-269.

3 Champion J.V., North P.F. (1968) Variation of flow birefringence with temperature in some liquid n-alkanes, Trans. Faraday Soc. 64, 238-733.

4 Cates M.E., Milner S.T. (1989) Role of shear in the isotropic-tolamellar transition, Phys. Rev. Lett. 62, 1856-1859.

5 Schmitt V., Lequeux F., Pousse A., Roux D. (1994) Flow behavior and shear induced transition near an isotropic/nematic transition in equilibrium polymers, Langmuir 10, 955-961.

6 Berret J.F., Roux D.C., Porte G., Lindner P. (1994) Shearinduced isotropic-to-nematic phase transition in equilibrium polymers, Europhys. Lett. 25, 521-526.

7 Decruppe J.P., Cressely R., Makhloufi R., Cappelaere E. (1995) Flow birefringence experiments showing a shear-banding structure in a CTAB solution, Colloid Polym. Sci. 273, 346-351.

8 Olmsted P.D., Lu C.-Y.D. (1997) Coexistence and phase separation in sheared complex fluids, Phys. Rev. E 56, 55-4415.

9 Cates M.E., Fielding S.M. (2006) Rheology of giant micelles, Adv. Phys. 55, 799-879.

10 Hess S. (1976) Flow alignment and flow-induced phase transition in liquid crystals, Z. Naturforsch. A 31, 1507-1513.

11 Olmsted P.D., Goldbart P.M. (1990) Theory of the nonequilibrium phase transition for nematic liquid crystals under shear flow, Phys. Rev. A 41, 4578-4581.

12 Pujolle-Robic C., Noirez L. (2001) Observation of shearinduced nematic-isotropic transition in side-chain liquid crystal polymers, Nature 409, 167-171.

13 Reys V., Dormoy Y., Gallani J.L., Martinoty P., Le Barny P., Dubois J.C. (1988) Short-range-order effects in the isotropic phase of a side-chain polymeric liquid crystal, Phys. Rev. Lett. 61, 2340-2343.

14 Noirez L., Baroni P., Mendil-Jakani H. (2009) The missing parameter in rheology: hidden solid-like correlations in viscous liquids, polymer melts and glass formers, Polymer International 58, 962-968.

15 Noirez L., Baroni P. (2010) Revealing the solid-like nature of glycerol at ambient temperature, J. Mol. Struct. 972, 16-21.

16 Noirez L., Mendil-Jakani H., Baroni P. (2011) Identification of finite shear-elasticity in the liquid state of molecular (OTP) and polymeric glass formers (PBuA), Philos. Mag. 91, 1977-1986.

17 Mendil H., Baroni P., Grillot I., Noirez L. (2006) Frozen states in the isotropic phase of liquid-crystal polymers, Phys. Rev. Lett. 96, 077801-3.

18 Noirez L., Baroni P. (2012) Identification of a low-frequency elastic behaviour in liquid water, J. Phys.: Condens. Matter 24, 372101-6.

19 Kahl P., Baroni P., Noirez L. (2013) Hidden solidlike properties in the isotropic phase of the 8CB liquid crystal, Phys. Rev. E 88, 50501-5.

20 Noirez L. (2005) Origin of shear-induced phase transitions in melts of liquid-crystal polymers, Phys. Rev. E 72, 051701-5.
21 Mendil H., Baroni P., Noirez L. (2005) Unexpected giant elasticity in side-chain liquid crystal polymer melts: a new approach for the understanding of shear induced phase transitions, Europhys. Lett. 72, 982-989.

22 Hertel G., Hoffmann H. (1988) Lyotropic nematic phases of double chain surfactants, Prog. Colloid. Polym. Sci. 76, 123-131.

23 Mendil-Jakani H., Baroni P., Noirez L. (2009) Shear-induced isotropic to nematic transition of liquid-crystal polymers: identification of gap thickness and slipping effects, Langmuir 25, 9, 5248-5252.

24 Noirez L., Baroni P., Cao H. (2012) Identification of shear elasticity at low frequency in liquid $n$-heptadecane, liquid water and RT-ionic liquids [emim][Tf2N], J. Mol. Liq. 176, 71-1986.

25 Mendil H., Baroni P., Baroni P. (2006) Solid-like rheological response of non-entangled polymers in the molten state, Eur. Phys. J. E 19, 77-87.

26 Noirez L., Mendil-Jakani H., Baroni P. (2009) New light on old wisdoms on molten polymers: conformation, slippage and shear banding in sheared entangled and unentangled melts, Macromol. Rapid Commun. 30, 1709-1714.

27 Metivier C., Rharbi Y., Magnin A., Bou Abboud A. (2012) Stick-slip control of the Carbopol microgels on polymethyl methacrylate transparent smooth walls, Soft Matt. 8, 7365-7367.

28 Chennevière A., Drockenmuller E., Damiron D., Cousin F., Boué F., Restagno F., Léger L. (2013) Quantitative analysis of interdigitation kinetics between a polymer melt and a polymer brush, Macromol. 46, 6955-6962.

29 Heidenreich S., Ilg P., Hess S. (2007) Boundary conditions for fluids with internal orientational degrees of freedom: apparent velocity slip associated with the molecular alignment, Phys. Rev. E 75, 66302-13.

30 Zaccone A., Blundell J.R., Terentjev E. (2011) Network disorder and nonaffine deformations in marginal solids, Phys. Rev. B 84, 174119-11.

31 Smallenburg F., Fillion L., Sciortoni F. (2014) Erasing noman's land by thermodynamically stabilizing the liquid-liquid transition in tetrahedral particles, Nat. Phys. 10, 653-657.

32 Schoen M., Hess S., Diestler D.J. (1995) Rheological properties of confined thin films, Phys. Rev. E 52, 2587-2602.

33 Volino F. (1997) Théorie visco-élastique non-extensive, Ann. Phys. Fr. 22, 181-231.

34 Manning M.L., Foty R.A., Steinberg M.S., Schoetz E.-M. (2010) Coaction of intercellular adhesion and cortical tension specifies tissue surface tension, PNAS 107, 12517-22.

35 Kahl P., Baroni P., Noirez L. (2016) Bringing to light hidden elasticity in the liquid state using in-situ pretransitional liquid crystal swarms, PLoS One 11, 2, e0147914.

Manuscript submitted in November 2016

Manuscript accepted in January 2017 Published online in March 2017

Cite this article as: L. Noirez (2017). Importance of Interfacial Interactions to Access Shear Elasticity of Liquids and Understand Flow Induced Birefringence from Liquid Crystals to Worm-Like Micellar Solutions, Oil Gas Sci. Technol 72, 10. 